\title{
A polymer chain trapped between two parallel repulsive walls: A Monte-Carlo test of scaling behavior
}

\author{
A. Milchev ${ }^{1, a}$ and K. Binder ${ }^{2, b}$ \\ 1 Institute for Physical Chemistry, Bulgarian Academy of Sciences, 1113 Sofia, Bulgaria \\ ${ }^{2}$ Institut für Physik, Johannes Gutenberg-Universität Mainz 55099 Mainz, Staudinger Weg 7, Germany
}

Eur. Phys. J. B 3, 477-484 (1998)

F. Schlesener kindly pointed out that in the scaling plot for the force, $D f$, versus $D / R_{g b}$ in our Figure 4 , the data was plotted with the wrong set of values for the radius of gyration $R_{g b}$. The $D f$ data points have been plotted erroneously versus $D / \sqrt{R_{g b}}$, rather than versus $D / R_{g b}$. In Table 1 the value for $f$ at $D=16$ and $N=512$ is $f=0.327$ rather than the mistyped value of 0.917 .

For completeness we give below the values of the mean squared gyration radii $R_{g b}$ of single unperturbed polymer chains of length $N$ which were used in our simulations (A. Milchev, K. Binder, Eur. Phys. J. B 3, 477 (1998)).

\begin{tabular}{|c|c|c|c|c|c|}
\hline $\mathrm{N}$ & 32 & 64 & 128 & 256 & 512 \\
\hline$R_{g b}^{2}$ & 6.31 & 14.59 & 34.74 & 78.44 & 178.3 \\
\hline
\end{tabular}

When replotted versus the correct ration $D / R_{g b}$, Figure 4 reveals a better agreement with the predicted scaling behavior of $D f \propto\left(\frac{D}{R_{g b}}\right)^{-\frac{1}{\nu}}$.

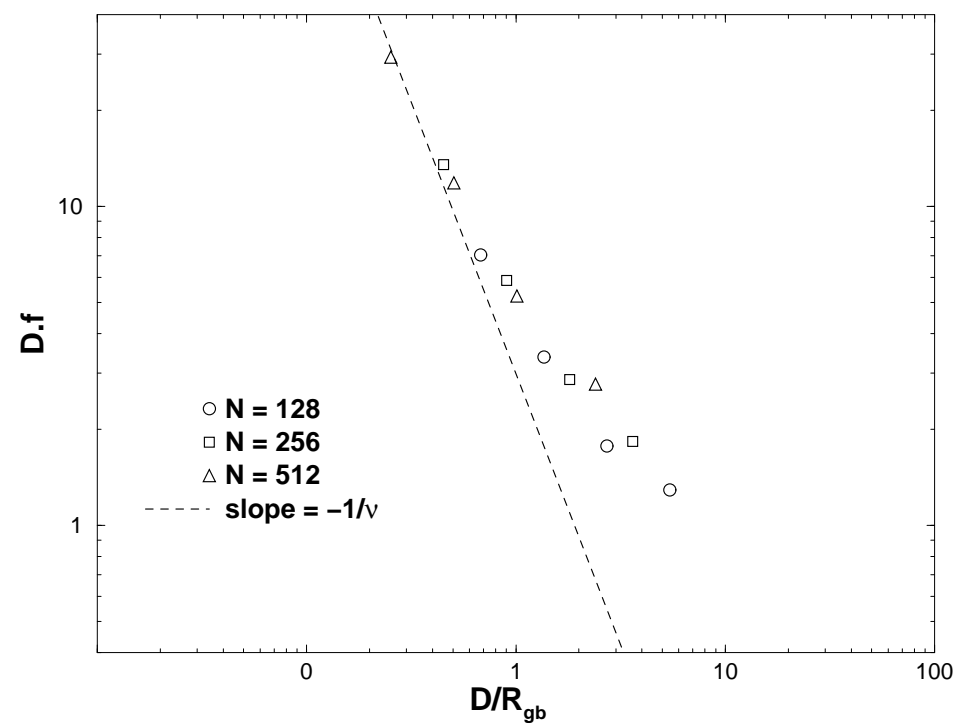

Fig. 4. Scaling plot for the force, $D f$ versus $D / R_{g b}$. The dashed straight line indicates the slope that the scaling function should exhibit for small $D / R_{g b}$.

a e-mail: milchev@plato.physik.uni-mainz.de

b e-mail: binder@chaplin.physik.uni-mainz.de 